\title{
Desenvolvimento motor de bebês em intervenção parental durante a puericultura: série de casos
}

\author{
Infants motor development in parental intervention during childcare: case series \\ Desarrollo motor de bebés en intervención parental durante la puericultura: serie de casos \\ Katia Virgínia Viana Cardoso', Cinthia Marques de Carvalho², Tayná Albuquerque Tabosa ${ }^{3}$, \\ Letícia Helene Mendes Ferreira ${ }^{4}$, Marcela de Castro Ferracioli Gama ${ }^{5}$
}

\begin{abstract}
RESUMO I O desenvolvimento infantil deve ser promovido pela família com apoio formal na puericultura. O presente estudo analisou o desempenho motor de bebês submetidos à intervenção parental na puericultura. Foi realizado um estudo de série de casos, observacional, longitudinal e descritivo, com 215 bebês de 0-18 meses atendidos em uma Unidade de Atenção Primária em Saúde de uma capital do Nordeste brasileiro. O desempenho motor dos bebês foi avaliado pela Alberta infant motor scale e orientações foram transmitidas aos pais a depender do atraso motor observado. Observouse que $77,7 \%$ dos bebês tiveram seu primeiro atendimento no primeiro semestre de vida, 57,6\% apresentaram baixo risco e $76,7 \%$ desempenho motor normal. Houve relação significativa entre a estratificação de risco e a idade gestacional e entre a estratificação de risco e o desempenho motor. Os intervalos entre as avaliações variaram de 7-252 dias. A porcentagem de bebês com atrasos motores é maior para os que chegam à puericultura após o terceiro trimestre de vida. Todos os bebês identificados com suspeita de atraso ou como atípicos, que frequentaram a puericultura, melhoraram o desempenho motor até a terceira avaliação. Bebês que são levados precocemente à puericultura, para orientações sobre intervenção parental, recuperam o atraso do desenvolvimento motor, porém muitas famílias não comparecem ao seguimento da puericultura ou chegam tardiamente para atendimento.
\end{abstract}

Descritores | Atividade Motora; Desenvolvimento Infantil; Intervenção Parental; Relatos de Casos.

\begin{abstract}
I Child development should be promoted by the family with formal support from childcare services. This study aims to analyze the motor performance of infants submitted to parental intervention in childcare. This is an observational, longitudinal and descriptive case series study with 215 infants aged 0-18 months, who were cared for at a Primary Health Care Unit in a Brazilian Northeastern capital. The infants' motor performance was assessed by the Alberta infant motor scale and instructions were transmitted to parents according to the observed motor delay. We observed that $77.7 \%$ of the infants had their first appointment in their first semester of life, 57.6\% had low risk and $76.7 \%$ had normal motor performance. Significant relationships between risk stratification and gestational age and between risk stratification and motor performance were found. The intervals between evaluations ranged from 7-252 days. A higher percentage of infants with motor delays was found in those who were taken to childcare after the third trimester of life. All infants who attended childcare and were identified with suspected delay or atypical development improved motor performance by the third evaluation. Infants who are taken to childcare early, for guidance on parental intervention, recover from motor development delay, but most families either do not attend childcare follow-up, or are long overdue for their appointment.
\end{abstract}

Keywords I Motor Activity; Child Development; Parental Intervention; Case Reports.

\footnotetext{
UUniversidade Federal do Ceará (UFC) - Fortaleza (CE), Brasil. E-mail: katiavirginia@ufc.br. Orcid: 0000-0002-6552-7124. ${ }^{2}$ Prefeitura Municipal de Fortaleza - Fortaleza (CE), Brasil. E-mail: cinthia.m@hotmail.com. Orcid: 0000-0001-7453-3051. 3Universidade Federal do Ceará (UFC) - Fortaleza (CE), Brasil. E-mail: taynaalbuquerquet@gmail.com. Orcid: 0000-0002-3964-081X. ${ }^{4}$ Universidade Federal do Ceará (UFC) - Fortaleza (CE), Brasil. E-mail: letyshelene@hotmail.com. Orcid: 0000-0002-6344-2020. ${ }^{5}$ Universidade Federal do Ceará (UFC) - Fortaleza (CE), Brasil. E-mail: marcelaferracioli@ufc.br. Orcid: 0000-0003-1782-691X. 
RESUMEN I El desarrollo infantil debe ser promovido por la familia con apoyo formal en la puericultura. Este estudio analizó el desempeño motor de bebés sometidos a intervención parental en la puericultura. Se realizó un estudio de serie de casos, observacional, longitudinal y descriptivo, con 215 bebés de 0-18 meses de edad que recibían atención en una Unidad de Atención Primaria de Salud de una capital del Nordeste de Brasil. El desempeño motor de los bebés se evaluó mediante la escala motora infantil de Alberta y se suministró información a los padres cuando se observó retraso motor en los bebés. El 77,7\% de los bebés recibieron su primer cuidado en el primer semestre de vida, el 57,6\% tuvieron bajo riesgo, y el 76,7\% presentaron un desempeño motor normal. Hubo una relación significativa entre la estratificación del riesgo y la edad gestacional, así como entre la estratificación del riesgo y el desempeño motor. Los intervalos entre evaluaciones oscilaron de 7 a 252 días. El porcentaje de bebés con retrasos motores es mayor para los que llegan a la puericultura después del tercer trimestre de vida. Todos los bebés identificados con sospecha de retraso o como atípicos, que asistieron a la puericultura, tuvieron una mejora de su desempeño motor hasta la tercera evaluación. Los bebés que se llevan precozmente a la puericultura para orientación sobre la intervención parental recuperan el retraso en el desarrollo motor, pero muchas familias no asisten al seguimiento de la puericultura o llegan tardíamente en esta atención.

Palabras clave | Actividad Motora; Desarrollo Infantil; Intervención Parental; Informes de Casos.

\section{INTRODUÇÃO}

O desenvolvimento infantil pode ser definido como um processo multidimensional e integral que engloba o crescimento físico, desenvolvimento motor, cognitivo e psicossocial nos primeiros anos de vida ${ }^{1}$. Quando a criança é exposta à fatores de risco biológico e/ou ambiental ela pode apresentar atrasos no desenvolvimento ${ }^{2}$.

Estimativas indicam que, em países de baixa e média renda, cerca de $43 \%$ das crianças de até 5 anos de idade não atingem seu potencial de desenvolvimento devido à exposição a riscos biológicos, psicossociais e ambientais ${ }^{2,3}$. Sabe-se que alguns déficits instalados na infância podem tornar-se problemas complexos com o passar dos anos, culminando em maior carência de recursos pessoais, econômicos e sociais para sua resolução, o que assinala para a necessidade de intervenções precoces ${ }^{4}$.

O acompanhamento do desenvolvimento infantil nos primeiros anos de vida é tarefa essencial para promoção à saúde, prevenção de agravos e identificação de atraso neuropsicomotor ${ }^{5}$. Dentre as práticas utilizadas para a melhora do neurodesenvolvimento está a intervenção parental (IP). As práticas parentais estão relacionadas ao processo de cuidado e socialização dos filhos por parte dos pais e cuidadores ${ }^{6}$ e têm base na teoria bioecológica ${ }^{7}$, a qual afirma que o desenvolvimento é influenciado pela interação da criança com o ambiente mais próximo dela, em que se encontra a família.

$\mathrm{Na}$ IP, os pais são orientados a promover um ambiente mais rico em estímulos para os filhos ${ }^{8}$. Orientações que incentivam, por exemplo, os pais a colocarem os bebês por mais tempo no chão e estimularem a postura de quatro apoios ${ }^{9}$, assim como treinamento prévio e suporte informacional aos pais ${ }^{10}$ levaram a resultados positivos para o desenvolvimento motor de bebês.

Recentemente, cerca de $10 \%$ das crianças menores de 6 anos do estado do Ceará apresentaram atrasos em, pelo menos, um domínio do desenvolvimento infantil ${ }^{11}$. Os autores sugerem que intervenções integradas, que considerem os fatores de risco e a desigualdade relacionada à pobreza, podem reduzir a prevalência de crianças com atrasos no desenvolvimento. Resta-nos saber se práticas voltadas para o empoderamento da família em relação ao desenvolvimento infantil, como a IP, podem promover o desenvolvimento motor adequado ao longo do tempo. Este estudo objetivou analisar o desempenho motor de bebês submetidos à IP na puericultura.

\section{METODOLOGIA}

Estudo observacional, longitudinal e descritivo, do tipo série de casos. Participaram 215 bebês de 0-18 meses (109 meninas), atendidos na puericultura de uma Unidade de Atenção Primária a Saúde (UAPS) de Fortaleza, capital cearense. A UAPS está localizada na Secretaria Executiva Regional III da cidade, que compreende 17 bairros da capital caracterizados como de subdesenvolvimento econômico em comparação aos bairros de outras secretarias regionais (IDH $0,38)^{12}$. A maioria dos bebês teve estratificação de risco da saúde avaliada pelo médico ou enfermeiro da Estratégia de Saúde da Família, segundo as diretrizes clínicas para atenção à criança do município ${ }^{13}$. A estratificação seguiu os seguintes critérios: 
No baixo risco estão incluídas crianças potencialmente livres de riscos com pré-natal sem intercorrências; aleitamento materno exclusivo até 6 meses de idade; peso ao nascer $\geq 2,5 \mathrm{~kg}$; apgar $\geq 8$, no $5^{\circ} \mathrm{min}$; sem reconhecimento de patologia específica, bom suporte familiar; mãe com mais de 8 anos de estudo, vacina em dia; recém-nascido $(\mathrm{RN})$ com triagem neonatal realizada.

No médio risco estão incluídas as crianças que apresentam vulnerabilidade determinada pela presença de vários fatores negativos, como: Prematuro limítrofe (37 semanas); mãe soropositiva para HIV, toxoplasmose, sífilis, hepatite $B$, com criança negativa para estas patologias; filho de mãe deficiente mental, doença mental ou transtornos psiquiátricos leves; morte materna; filho de mãe com menos de 20 anos e mais de três partos; história de óbitos de menores de cinco anos no núcleo familiar da criança; criança manifestamente indesejada; criança menor de 6 meses de idade que não se encontra em aleitamento materno exclusivo, mãe com escolaridade de 3 a 7 anos e 11 meses de estudo; filho de mãe com menos de 15 anos ou mais de 40 anos e/ou solteira; filho de mãe sem suporte familiar; crianças que vivem em situação de risco e vulnerabilidade; chefe de família sem fonte de renda; filho de mãe de etnia indígena; vacina atrasada; $\mathrm{RN}$ sem triagem neonatal.

No alto risco estão incluídas as crianças que se encontram submetidas a fatores de risco oriundos dos campos de saúde, porém, de forma mais intensa e de maior gravidade do que no grupo anterior.

Os bebês foram incluídos no estudo de forma consecutiva e aleatória seguindo os critérios de inclusão: autorização dos pais através do termo de consentimento livre e esclarecido e apresentar prontuário na UAPS e exclusão: apresentar afecções osteomioarticulares ou infecções com febre e participação em programas de intervenção.

Para avaliação do desempenho motor dos bebês foi utilizada a Alberta infant motor scale (AIMS) ${ }^{14,15}$. A AIMS é composta por 58 itens motores organizados em quatro subescalas: prono(21), supino(9), sentado(12) e em pé(16). A pontuação total é obtida pela soma dos escores das subescalas, variando de 0-58. Além disso, foram utilizados tatame e brinquedos durante essa avaliação. A avaliadora foi uma fisioterapeuta, com capacitação específica e experiência de 2 anos de aplicação da AIMS. Ela manuseava minimamente o bebê e observava a movimentação da criança em cada uma das posições, levando em consideração a superfície do corpo que sustenta o peso, postura e movimentos antigravitacionais, durante 20-30 minutos.

Ao término da avaliação, a pontuação foi convertida em percentis do desempenho motor da criança, podendo variar de $<5^{\circ}$ ao $90^{\circ}$. O desempenho motor dos bebês foi classificado como atípico com percentil $<5 \%$, de 10 $25 \%$ com suspeita de atraso e $50-90 \%$ como normal $^{14}$. A população estudada nesta pesquisa foi de crianças atendidas na puericultura e sujeitas a risco psicossocial, portanto, optamos pela classificação apresentada por Piper et al. (1992) para a população canadense, para que as crianças atípicas fossem encaminhadas para o serviço de referência e as com suspeita de atraso fossem acompanhadas na puericultura com intervenção parental, prática que tem como objetivo minimizar as práticas maternas inadequadas que possam contribuir com o atraso no desenvolvimento.

De acordo com o resultado da avaliação motora, a fisioterapeuta realizou intervenções com os pais através de orientações e demonstrações de posicionamentos e brincadeiras para estabelecer estratégias da IP a ser realizada em casa. Os estímulos sugeridos foram: ampliar as vivências no chão, proporcionar estímulos com objetos coloridos, pedir para a criança pegar um objeto em quatro apoios, caminhar lateralmente com apoio, estímulos de posição de supino para prono, de prono para sentado, de sentado para de pé, de pé para marcha lateral, estímulos proprioceptivos com objetos de diferentes texturas, formatos e ambientes diferentes (areia, colchão, solo). Os pais eram orientados a retornar mensalmente para a UAPS para realizar a avaliação e o acompanhamento do desempenho motor dos bebês.

Foi realizada análise descritiva dos dados com frequência absoluta e relativa de bebês avaliados, idade na primeira avaliação, idade gestacional, estratificação de risco, desempenho motor e quantidade de avaliações realizadas por bebê. Foram apresentados média e desvio padrão da pontuação alcançada pelos bebês na AIMS e do intervalo entre as avaliações realizadas por bebê.

$\mathrm{O}$ teste qui-quadrado $\left(\mathrm{X}^{2}\right)$ de Pearson foi utilizado para relacionar as variáveis nominais e dicotômicas: idade gestacional, estratificação de risco e desempenho motor. O teste T de Student one-sample foi utilizado para comparar a média do desempenho motor na AIMS com o desempenho esperado para a população brasileira ${ }^{15}$. O nível de significância adotado foi $\mathrm{p}<0,05$.

\section{RESULTADOS}

Da série de casos, $77,7 \%(\mathrm{n}=167)$ dos bebês foram atendidos pela primeira vez na puericultura entre 0-179 dias de vida, $16,3 \%(n=35)$ entre $180-359$ dias de vida e 
6\%( $\mathrm{n}=13)$ com mais de 360 dias de vida. Dessa série de casos, 20,4\%(n=44) não apresentavam estratificação de risco em seus prontuários, $57,6 \%(\mathrm{n}=124)$ apresentaram baixo risco, $2,7 \%(\mathrm{n}=16)$ médio e $19,0 \%(\mathrm{n}=41)$ alto.

Quanto à idade gestacional, 13\%(n=28) dos bebês foram classificados como pré-termo ( $<37$ semanas). Houve relação significativa entre a estratificação de risco e a idade gestacional $\left(\mathrm{X}^{2}=32,32 ; \mathrm{p}<0,001\right)$, sendo que $53,5 \%(\mathrm{n}=15)$ dos bebês pré-termo apresentaram alto risco, $7,1 \%(\mathrm{n}=2)$ médio, $17,8 \%(\mathrm{n}=5)$ baixo e em $21,4 \%(n=6)$ não foi registrada a estratificação de risco. Além disso, 75\%(n=21) dos bebês pré-termo apresentaram desempenho motor normal, $14,3 \%(n=4)$ com suspeita de atraso e $10,7 \%(\mathrm{n}=3)$ atípico.

$\mathrm{Na}$ primeira avaliação, $76,7 \%(\mathrm{n}=165)$ dos bebês apresentaram desempenho motor normal, $19,5 \%(n=42)$ com suspeita de atraso e 3,7\%(n=8) atípico (Figura1). Houve relação significativa entre a estratificação de risco e o desempenho motor $\left(X^{2}=17,62 ; p=0,001\right)$, sendo que dos bebês com suspeita de atraso, 4,7\%(n=2) apresentaram alto risco, $9,5 \%(\mathrm{n}=4)$ médio, $59,5 \%(\mathrm{n}=25)$ baixo e no restante não foi registrada a estratificação de risco. Já os bebês que apresentaram desempenho motor atípico, $37,5 \%(n=3)$ apresentaram alto risco, $25 \%(n=2)$ baixo risco e o restante não foi registrada a estratificação de risco.

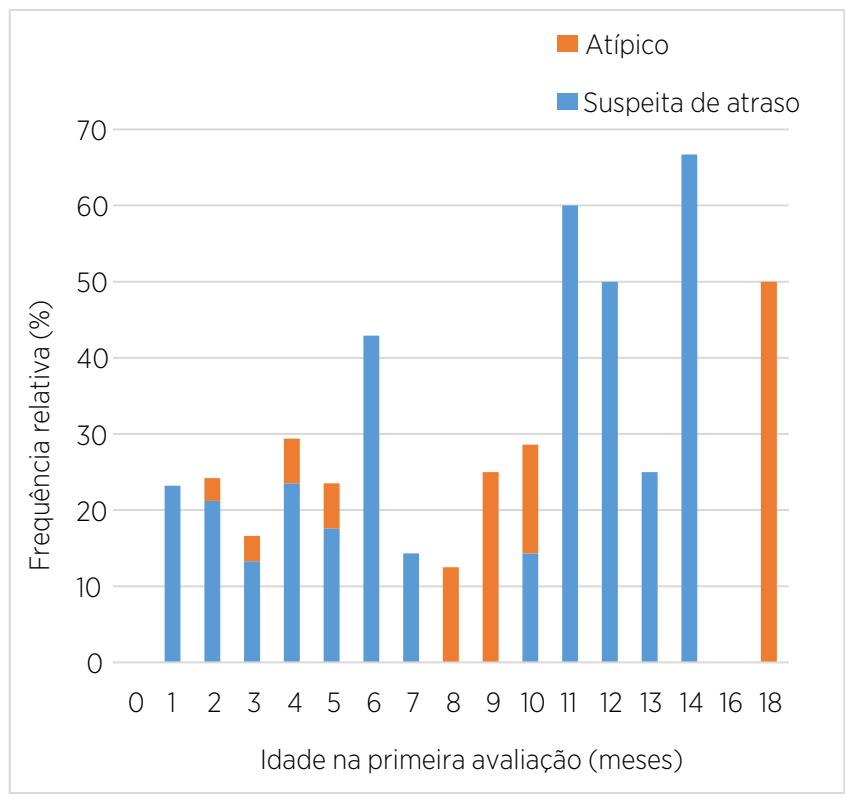

Figura 1. Porcentagem de bebês com desempenho motor com suspeita de atraso e atípico na primeira avaliação

De todos os bebês, $49,3 \%(n=106)$ foram avaliados uma única vez, 22,3\%(n=48) duas vezes, $13,9 \%(\mathrm{n}=30)$ três vezes, $6,5 \%(n=14)$ quatro vezes e $7,9 \%(n=17)$ cinco vezes. $\mathrm{O}$ intervalo médio entre a primeira e a segunda avaliações foi $64,7 \pm 36,5$ (mínimo 7 e máximo 277 ) dias, entre a segunda e a terceira foi 70,6 $\pm 44,9$ (mínimo 15 e máximo 252) dias, entre a terceira e a quarta foi $64,1 \pm 33,7$ (mínimo 16 e máximo 187) dias e entre a quarta e a quinta foi 56,0 31,5 (mínimo 23 e máximo 191) dias. Dos bebês avaliados apenas uma vez, 21,7\%(n=23) apresentaram desempenho motor com suspeita de atraso e 2,8\%(n=3) atípico. A Figura 2 mostra a quantidade de bebês submetidos à duas ou mais avaliações com a AIMS na puericultura e a classificação do desempenho motor.

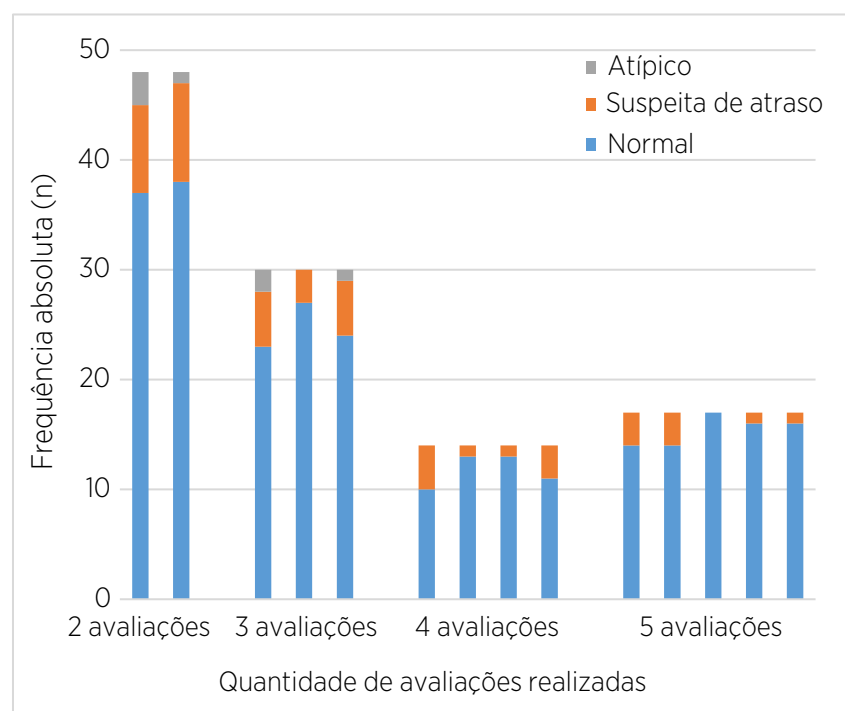

Figura 2. Número de bebês com desempenho motor normal, com suspeita de atraso e atípico de acordo com a quantidade de avaliações na puericultura

Dos bebês identificados com desempenho motor com suspeita de atraso ou atípico na primeira avaliação, $48 \%(n=24)$ voltaram para atendimento na UAPS e, destes, $62,5 \%(n=15)$ melhoraram o desempenho motor nesta segunda avaliação. Dos bebês que ainda mantiveram o desempenho motor com suspeita de atraso ou atípico na segunda avaliação $(n=9)$, apenas $44,4 \%(n=4)$ voltaram ao atendimento na UAPS para realizar a terceira avaliação e todos melhoraram o desempenho motor neste momento.

Os resultados da pontuação total dos bebês na AIMS, na primeira avaliação, e da comparação desta pontuação com a esperada para a população brasileira mostraram que bebês com 1,3,5 e 7 meses atendidos na puericultura apresentaram desempenho motor superior ao esperado para a população brasileira. Bebês com 6, 9, 11, 12, 14 e 18 meses apresentaram desempenho motor inferior ao esperado para a população brasileira, mas estas diferenças não foram significativas (Tabela 1 ). 
Tabela 1. Desempenho motor na AIMS dos bebês atendidos na puericultura comparado com o desempenho motor esperado para a população brasileirat ${ }^{15}$

\begin{tabular}{|c|c|c|c|}
\hline $\begin{array}{l}\text { Idade } \\
\text { (meses) }\end{array}$ & $M \pm D P$ & 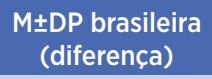 & $\begin{array}{l}\text { Teste T } \\
(p)\end{array}$ \\
\hline $0-<1$ & $4,9 \pm 1,0$ & $4,3 \pm 1,2(0,5)$ & 0,15 \\
\hline $1-<2$ & $6,9 \pm 1,4$ & $5,7 \pm 1,3(1,2)$ & 0,00 \\
\hline $2-<3$ & $9,4 \pm 2,3$ & $8,8 \pm 2,4(0,6)$ & 0,15 \\
\hline $3-<4$ & $13,2 \pm 2,7$ & $11,5 \pm 3,4(1,6)$ & 0,02 \\
\hline $4-<5$ & $16,8 \pm 3,8$ & $15,0 \pm 3,9(1,7)$ & 0,13 \\
\hline $5-<6$ & $25,2 \pm 5,9$ & $19,4 \pm 5,5(5,7)$ & 0,02 \\
\hline $6-<7$ & $21,7 \pm 3,3$ & $23,9 \pm 7,6(-1,4)$ & 0,46 \\
\hline $7-<8$ & $38,7 \pm 5,6$ & $30,4 \pm 6,5(8,2)$ & 0,03 \\
\hline $8-<9$ & $39,3 \pm 7,2$ & $35,7 \pm 8,9(3,5)$ & 0,39 \\
\hline $9-<10$ & $34,3 \pm 8,8$ & $39,6 \pm 8,6(-5,3)$ & 0,39 \\
\hline $10-<11$ & $48,1 \pm 5,9$ & $44,1 \pm 8,1(3,9)$ & 0,20 \\
\hline $11-<12$ & $47,2 \pm 5,0$ & $48,9 \pm 5,4(-1,7)$ & 0,54 \\
\hline $12-<13$ & $48,3 \pm 4,4$ & $53,4 \pm 3,5(-5,1)$ & 0,21 \\
\hline $13-<14$ & $55,7 \pm 3,1$ & $54,5 \pm 3,5(1,0)$ & 0,69 \\
\hline $14-<15$ & $54,5 \pm 2,0$ & $56,0 \pm 3,1(-1,5)$ & 0,37 \\
\hline $15-<16$ & - & $57,0 \pm 2,0$ & - \\
\hline $16-<17$ & $58,0 \pm 0,0$ & $57,7 \pm 0,7(0,2)$ & * \\
\hline $17-<18$ & - & $57,7 \pm 0,8$ & - \\
\hline $18-<19$ & $36,0 \pm 22,0$ & $57,8 \pm 0,4(-21,8)$ & 0,50 \\
\hline
\end{tabular}

Fonte: M: Média. DP: Desvio Padrão. *não aplicável $(n=1)$.

\section{DISCUSSÃO}

O estudo analisou o desempenho motor de bebês submetidos à IP na puericultura de uma UAPS de Fortaleza/ CE. Observou-se que houve aumento do número de bebês com desempenho motor normal da primeira para a segunda avaliação e da segunda para a terceira, indicando a relevância da IP e do seguimento na puericultura para o desenvolvimento motor. Apesar disso, ressalta-se a dificuldade de seguimento do atendimento, tendo em vista que quase metade da amostra não voltou para realização das ações da puericultura.

Além disso, cerca de $22 \%$ dos bebês tiveram seu primeiro atendimento na puericultura somente após o primeiro semestre de vida, o que chama a atenção para a possibilidade de atrasos já estarem acontecendo previamente, sem que possíveis intervenções pudessem ser desenvolvidas adequadamente e em tempo hábil para mudanças. Esta hipótese foi proposta diante dos dados (Figura 1) que apontam para maior frequência de bebês identificados com desempenho motor com suspeita de atraso ou atípicos ao serem avaliados pela primeira vez somente após o primeiro semestre de vida. Apesar de não termos resultados e estudos que testem essa hipótese, alguns estudos têm mostrado que bebês submetidos à IP antes dos 6 meses de vida apresentam melhoras no desempenho motor ${ }^{10,16}$.
Com relação ao seguimento na puericultura, alguns estudos mostraram que mais de $90 \%$ das crianças nordestinas investigadas frequentaram a puericultura e cerca de dois terços delas tiveram pelo menos 7 acompanhamentos ${ }^{17,18}$. A prevalência de crianças nordestinas com puericultura incompleta foi de 53,6\% ${ }^{19}$. Quando comparado a outras regiões brasileiras, o Nordeste exibe fatores que podem estar associados à dificuldade de acompanhamento, como grande território geográfico, tamanho populacional e status econômico da região. Portanto, estas crianças estão mais propensas a não terem acompanhamento de saúde completo ${ }^{19}$.

A identificação precoce de atrasos no desenvolvimento motor na puericultura permite a realização de intervenções efetivas. A intervenção precoce pode ser capaz de melhorar a qualidade de vida das famílias que se encontram em situação de risco socioeconômico e promover o desenvolvimento infantil ${ }^{20}$. As experiências da criança, especialmente na primeira infância, são capazes de produzir mudanças permanentes no seu cérebro. Uma mudança no ambiente parece ter efeitos rápidos na anatomia do cérebro e menos de três semanas de exposição a um ambiente novo e complexo são suficientes para causar expansões cerebrais ${ }^{21}$. Assim também, a privação severa está associada a alterações negativas no volume e na estrutura do cérebro na idade adulta, mesmo quando indivíduos expostos a essa forma de privação foram posteriormente criados em famílias que proporcionavam ambientes favoráveis para o resto da infância ${ }^{22}$.

Uma dificuldade encontrada para o acompanhamento adequado destes bebês se refere ao preenchimento incompleto de registros. Isto é comumente justificado pelos profissionais devido ao tempo que é gasto com preenchimento de formulários, guias e justificativas, que chega a ser maior do que aquele dedicado aos pacientes ${ }^{23}$. Estes fatores representam, além de limitações ao presente estudo, grandes dificuldades no acompanhamento dos bebês, cujo objetivo central é orientar aos familiares quanto ao desenvolvimento da criança. Essas orientações quanto à importância da avaliação constante do crescimento e desenvolvimento infantil representam, para gestores e equipes de saúde, um momento oportuno para propor estratégias de promoção da saúde e prevenção de doenças ${ }^{23}$.

Os bebês desta série de casos não apresentaram desempenho motor na AIMS diferente do esperado para a população brasileira ${ }^{15}$. Bebês brasileiros apresentaram índice de desempenho motor 34,6\% abaixo do desempenho de bebês de outros países e essa diferença foi reduzida quando alcançaram 15 meses de idade ${ }^{24}$. Acredita-se que as diferenças de desempenho motor estão relacionadas 
às características culturais e sociais da população e que variações no ambiente criam e restringem possibilidades para ação. Um estudo mostrou que uma amostra brasileira apresentou baixas oportunidades de estimulação ambiental para o desenvolvimento motor e que famílias com melhores níveis econômicos apresentaram oportunidades mais favoráveis ${ }^{25}$.

A estratificação de risco foi relacionada com a idade gestacional e com o desempenho motor dos bebês. Assim, reforça-se que as desvantagens ambientais, somadas a fatores biológicos, são capazes de influenciar negativamente o desenvolvimento infantil ${ }^{2}$. O conhecimento sobre os fatores de risco que podem levar ao atraso do desenvolvimento é imprescindível para implementar recursos que visem diminuir seus efeitos sobre a criança e a família ${ }^{25}$.

O presente estudo apresenta limitações quanto à falta de informações em alguns prontuários e à falta de adesão e frequência das famílias dos bebês nos atendimentos da puericultura, gerando dificuldade de acompanhamento dos bebês e de análise do efeito da intervenção parental no desenvolvimento motor destes. Sugere-se que profissionais e serviços de atenção às famílias possam, além de continuar estimulando o seguimento na puericultura, propor estratégias de controle das informações e da IP que superem a necessidade de a família se deslocar à unidade de saúde (ex. através de telecomunicação e meio digital).

\section{CONCLUSÃO}

Nessa série de casos, o desempenho motor dos bebês foi influenciado pela estratificação de risco, idade gestacional e atendimento e seguimento na puericultura. $\mathrm{O}$ estudo avança na compreensão de que bebês que são levados o quanto antes à puericultura, para orientações sobre a IP, recuperam o atraso do desenvolvimento motor. Apesar disso, grande parte das famílias não comparece ou chega tardiamente para atendimento. Sugere-se que estratégias de empoderamento da família no cuidado e na promoção do desenvolvimento infantil, como orientações para IP em diversos formatos, devem ser utilizadas no âmbito da atenção à saúde.

\section{REFERÊNCIAS}

1. Papalia DE, Feldman RD. Desenvolvimento humano. São Paulo: Artmed; 2013.
2. Lima MC, Eickmann SH, Lima ACV, Guerra MQ, Lira PIC, Huttly SRA, et al. Determinants of mental and motor development at 12 months in a low income population: a cohort study in northeast Brazil. Acta Paediatr. 2004;93(7):969-75. doi: 10.1111/j.16512227.2004.tb18257.x.

3. Ferreira RC, Alves CRL, Guimarães MAP, Menezes KKP, Magalhães LC. Effects of early interventions focused on the family in the development of children born preterm and/or at social risk: a meta-analysis. J Pediatr (Rio J). 2020;96(1):20-38. doi: 10.1016/j.jped.2019.05.002.

4. Comitê Científico do Núcleo Ciência Pela Infância. Estudo nำ1: O impacto do desenvolvimento na primeira infância sobre a aprendizagem. São Paulo: Núcleo Ciência Pela Infância; 2014 [cited 2021 Jul. 29]. Available from: https://bit.ly/3rll7lk

5. Ministério da Saúde (BR). Secretaria de Atenção à Saúde (BR). Diretrizes de estimulação precoce: crianças de zero a 3 anos com atraso no desenvolvimento neuropsicomotor decorrente de microcefalia. Brasília, DF; 2016

6. Schmidt B, Staudt ACP, Wagner A. Intervenções para promoção de práticas parentais positivas: uma revisão integrativa. Contextos Clin. 2016;9(1):2-18. doi: 10.4013/ctc.2016.91.01.

7. Bronfenbrenner U, Morris PA. The ecology of developmental processes. In: Damon W, editor. Handbook of child psychology: theoretical models of human development. 5th ed. Hoboken: Wiley; 1997. p. 993-1028.

8. Pungello EP, Iruka IU, Dotterer AM, Mills-Koonce R, Reznick JS. The effects of socioeconomic status, race, and parenting on language development in early childhood. Dev Psychol. 2009;45(2):544. doi: 10.1037/a0013917.

9. Silva PL, Santos DCC, Gonçalves VMG. Influência de práticas maternas no desenvolvimento motor de lactentes do 6ㅇo 12ํㅜ mês de vida. Braz J Phys Ther. 2006;10(2):225-31. doi: 10.1590/ S1413-35552006000200014

10. Sá FE, Nunes NP, Gondim EJL, Almeida AKF, Alencar AJC, Cardoso KVV. Intervenção parental melhora o desenvolvimento motor de lactentes de risco: série de casos. Fisioter Pesqui. 2017;24(1):15-21. doi: 10.1590/1809-2950/15828624012017.

11. Correia LL, Rocha HAL, Sudfeld CR, Rocha SGMO, Leite AJM, Campos JS, et al. Prevalence and socioeconomic determinants of development delay among children in Ceará, Brazil: A population-based study. PloS one. 2019;14(11):e0215343. doi: 10.1371/journal.pone.0215343

12. Fortaleza. Portal de Transparência de Fortaleza: Dados Abertos [Internet]. Fortaleza: Secretaria Municipal de Finanças; 2010 [cited 2021 Jul. 29]. Available from: https://transparencia. fortaleza.ce.gov.br/

13. Fortaleza. Secretaria Municipal da Saúde de Fortaleza. Diretrizes Clínicas: Atenção à Criança [Internet]. Fortaleza: Secretaria Municipal da Saúde de Fortaleza; 2016 [cited 2021 Jul. 29]. Available from: https://bit.ly/3j3X4kj.

14. Piper MC, Pinnell LE, Darrah J, Maguire T, Byrne PJ. Construction and validation of the Alberta Infant Motor Scale (AIMS). Can J Public Health. 1992:83:46-50.

15. Saccani R, Valentini NC, Pereira KR. New Brazilian developmental curves and reference values for the Alberta Infant Motor Scale. Infant Behav Dev. 2016;45:38-46. doi: 10.1016/j. infbeh.2016.09.002. 
16. Formiga CKMR, Pedrazzani ES, Tudella E. Desenvolvimento motor de lactentes pré-termo participantes de um programa de intervenção fisioterapêutica precoce. Braz J Phys Ther. 2004;8(3):239-45.

17. Facchini LA, Piccini RX, Tomasi E, Thumé E, Silveira DS, Siqueira FV, et al. Desempenho do PSF no Sul e no Nordeste do Brasil: avaliação institucional e epidemiológica da Atenção Básica à Saúde. Cien Saude Colet. 2006;11(3):669-81. doi: 10.1590/ S1413-81232006000300015.

18. Piccini RX, Facchini LA, Tomasi E, Thumé E, Silveira DS, Siqueira FV, et al. Efetividade da atenção pré-natal e de puericultura em unidades básicas de saúde do Sul e do Nordeste do Brasil. Rev Bras Saude Mater Infant. 2007;7(1):75-82. doi: 10.1590/ S1519-38292007000100009.

19. Santos AS, Duro SMS, Cade NV, Facchini LA, Tomasi E. Access to child care services in the Northeast and in the South Regions of Brazil. Rev Bras Saude Mater Infant. 2017;17(3):447-60. doi: 10.1590/1806-93042017000300003.

20. Veldman SL, Santos R, Jones RA, Sousa-Sá E, Okely AD. Associations between gross motor skills and cognitive development in toddlers. Early Hum Dev. 2019;132:39-44. doi: 10.1016/j.earlhumdev.2019.04.005.
21. Scholz J, Allemang-Grand R, Dazai J, Lerch JP. Environmental enrichment is associated with rapid volumetric brain changes in adult mice. Neuroimage, 2015;109:190-8. doi: 10.1016/j. neuroimage.2015.01.027

22. Mackes NK, Golm D, Sarkar S, Kumsta R, Rutter M, Fairchild G, et al. Early childhood deprivation is associated with alterations in adult brain structure despite subsequent environmental enrichment. Proc Natl Acad Sci U S A. 2020;117(1):641-9. doi: 10.1073/pnas.1911264116.

23. Silva ICA, Rebouças CBA, Lúcio IML, Bastos MLA. Consulta de enfermagem em puericultura: uma realidade de atendimento. Rev Enferm UFPE on line. 2014;8(4):966-73. doi: 10.5205/1981-8963-v8i4a9767p966-973-2014.

24. Saccani R, Valentini NC. Análise transcultural do desenvolvimento motor de crianças brasileiras, gregas e canadenses avaliadas com a Alberta Infant Motor Scale. Rev Paul Pediatr. 2013;31(3):350-8. doi: 10.1590/S0103-05822013000300012.

25. Defilipo EC, Frônio JS, Teixeira MTB, Leite ICG, Bastos RR, Vieira MT, et al. Oportunidades do ambiente domiciliar para o desenvolvimento motor. Rev Saude Publica. 2012;46(4):633-41. doi: 10.1590/S0034-89102012005000040. 
A versão do artigo "Desenvolvimento motor de bebês em intervenção parental durante a puericultura: série de casos" publicado no volume 28, número 2, 2021, disponibilizada inicialmente continha erros em relação ao nome da autora.

Onde se lia:

Cinthia Marques de Carvalho ${ }^{3}$

Leia-se:

Tayná Albuquerque Tabosa ${ }^{3}$ 